\title{
Thermal hard-photons probing multifragmentation in nuclear collisions around the Fermi energy
}

\author{
D.G. D'Enterria ${ }^{a, b, *}$ ) And G. Martínez ${ }^{a, *}$ ) \\ FOR THE TAPS COLLABORATION \\ ${ }^{a}$ GANIL, BP 5027, 14076 Caen Cedex 5, France \\ ${ }^{b}$ Grup de Física de les Radiacions, Universitat Autònoma de Barcelona, \\ 08193 Cerdanyola, Catalonia
}

Received XXX

\begin{abstract}
Hard-photon $\left(\mathrm{E}_{\gamma}>30 \mathrm{MeV}\right)$ emission issuing from proton-neutron bremsstrahlung collisions is investigated in four different heavy-ion reactions at intermediate bombarding energies $\left({ }^{36} \mathrm{Ar}+{ }^{197} \mathrm{Au},{ }^{107} \mathrm{Ag},{ }^{58} \mathrm{Ni},{ }^{12} \mathrm{C}\right.$ at $\left.60 \mathrm{~A} \mathrm{MeV}\right)$ coupling the TAPS photon spectrometer with two charged-particle multidetectors covering more than $80 \%$ of the solid angle. The hard-photon spectra of the three heavier targets result from the combination of two distinct exponential distributions with different slope parameters, a result which deviates from the behaviour expected for hard-photon production just in first-chance proton-neutron collisions. The thermal origin of the steeper bremsstrahlung component is confirmed by the characteristics of its slope and angular distribution. Such thermal hard-photons convey undisturbed information of the thermodynamical state of hot and excited nuclear systems undergoing multifragmentation.
\end{abstract}

\section{Introduction}

The production of elementary particles not present in the nucleus as real particles (such as photons, mesons or dileptons) has attracted much interest in heavyion physics at intermediate bombarding energies $\left(20 \mathrm{~A} \mathrm{MeV} \leq E_{l a b} \leq 100 \mathrm{AMeV}\right)$ since they are precise probes of the phase-space evolution of nucleus-nucleus collisions [1]. Among the experimental probes, photons are leading observables because, at variance with particles and nuclear fragments, after their production they do not suffer any final-state (either Coulomb or strong) interaction with the surrounding medium, giving a faithful image of the emission source. Above $\mathrm{E}_{\gamma}$ $=30 \mathrm{MeV}$, a bulk of experimental and theoretical evidences indicate that photons are mainly produced during the first instants of the reaction in individual proton-neutron bremsstrahlung $(p n \gamma)$ collisions within the participant zone [1] 2]. Hard-photons reveal, hence, the preequilibrium conditions existing in the initial high-compression phase of the reaction. Recent experimental results collected with a second-generation of photon spectrometers such as the "Two Arm Photon Spectrometer" TAPS [3] coupled to charged-particle multidetectors covering a large fraction of the available phase-space, have allowed a more precise and exclusive analysis of hard-photon energy spectra, angular distributions and two-photon correlation functions in a wide photon energy range, with high statistics data sets and for different projectile-target combinations [4, 5]. These new measurements

*) Present address: SUBATECH, BP 20722, 44307 Nantes Cedex 3, France 
have pointed out that, aside from the pure first-chance (or "direct") $p n \gamma$ emission scenario, an additional hard-photon softer component (referred to as "thermal" hard-photons) shows up accounting for up to a third of the total hard-photon yield. Those thermal hard-photons are emitted in secondary $p n \gamma$ collisions in a later stage of the reaction when part of the available energy has been thermalized among intrinsic degrees of freedom [4, 6].

The interest of such an observation relies on the fact that thermal hard-photons would constitute a singular probe of the intermediate dissipative stages of the reaction where the process of nuclear fragmentation is supposed to take place, giving hints on both the time-scale of this process and, eventually, on the thermodynamical state (temperature, density, degree of thermalization ...) of the fragmenting source(s). This information is fundamental to understand the mechanism which drives the copious production of intermediate-mass-fragments (IMF, $3 \leq Z \leq 20$ ) experimentally observed in intermediate-energy heavy-ion collisions. Indeed, in the present-day there is no general agreement among the different experimental results and the different statistical [7, 8] or dynamical [9] theoretical models regarding the underlying physical mechanism behind such multifragmentation process (see e.g. [10]). The key issue in these studies is whether or not an equilibrated system is created in the course of the collision. This is essential to extract valid information on the thermodynamical properties of the highly excited nuclei produced in such reactions, and ultimately to signal the existence of a liquid-gas phase transition in finite nuclear systems.

To confirm the existence of thermal hard-photon emission and to study thoroughly this process, two experimental campaigns of the TAPS collaboration have been carried out in 1997 and 1998 at the KVI and GANIL 11] facilities coupling, for the first time, a photon spectrometer with two different charged-particle multidetectors covering more than $80 \%$ of $4 \pi$. Here we report some first results of hard-photon and fragment production in the ${ }^{36} \mathrm{Ar}+{ }^{197} \mathrm{Au},{ }^{107} \mathrm{Ag},{ }^{58} \mathrm{Ni},{ }^{12} \mathrm{C}$ reactions at $60 \mathrm{~A} \mathrm{MeV}$ studied at KVI.

The paper is organized as follows. Section 2 is devoted to the description of the experimental setup. The first inclusive and exclusive experimental results obtained and their interpretation are shown in Section 3. A summary is finally given in Section 4 .

\section{Experimental setup}

The experiment was performed end of 1997 at the KVI (Groningen) facility using the ${ }^{36} \mathrm{Ar}$ beams delivered by the $\mathrm{K}=600$ superconducting AGOR cyclotron at 37.1 MHz. Four systems were studied, ${ }^{36} \mathrm{Ar}+{ }^{197} \mathrm{Au},{ }^{107} \mathrm{Ag},{ }^{58} \mathrm{Ni},{ }^{12} \mathrm{C}$ at $60 \mathrm{~A} \mathrm{MeV}$, with different beam intensities (ranging from $3.0 \mathrm{nA}$ up to $12.5 \mathrm{nA}$ ) and target thicknesses (from $1 \mathrm{mg} / \mathrm{cm}^{2}$ to $18 \mathrm{mg} / \mathrm{cm}^{2}$ ). The goals of the campaign were to study exclusively photon production in a wide energetic domain, ranging from statistical 
Giant-Dipole-Resonance gamma-rays $\left(10 \mathrm{MeV} \leq E_{\gamma} \leq 20 \mathrm{MeV}\right)$ to very energetic hard-photons (up to $\mathrm{E}_{\gamma} \approx 200 \mathrm{MeV}$ ), in coincidence with multi-fragment emission processes for different heavy-ion systems. For that purpose, we used concurrently a wide dynamic range photon spectrometer, together with two phoswich multidetectors for light-charged-particles (LCP, $\mathrm{Z} \leq 2$ ) and intermediate-mass-fragments (IMF, $3 \leq \mathrm{Z} \leq 10$ ) detection (fig. 1]). TAPS electromagnetic calorimeter comprising $384 \mathrm{BaF}_{2}$ scintillation modules in a six-block configuration and covering a solid angle of about $15 \%$ of $4 \pi$ was used to measure the double differential cross section $d \sigma / d \Omega_{\gamma} d E_{\gamma}$ for photons of $10 \mathrm{MeV} \leq E_{\gamma} \leq 200 \mathrm{MeV}$. Photons were separated from charged particles and neutrons on the basis of pulse-shape analysis, time-of-flight and TAPS charged-particle veto information [12].

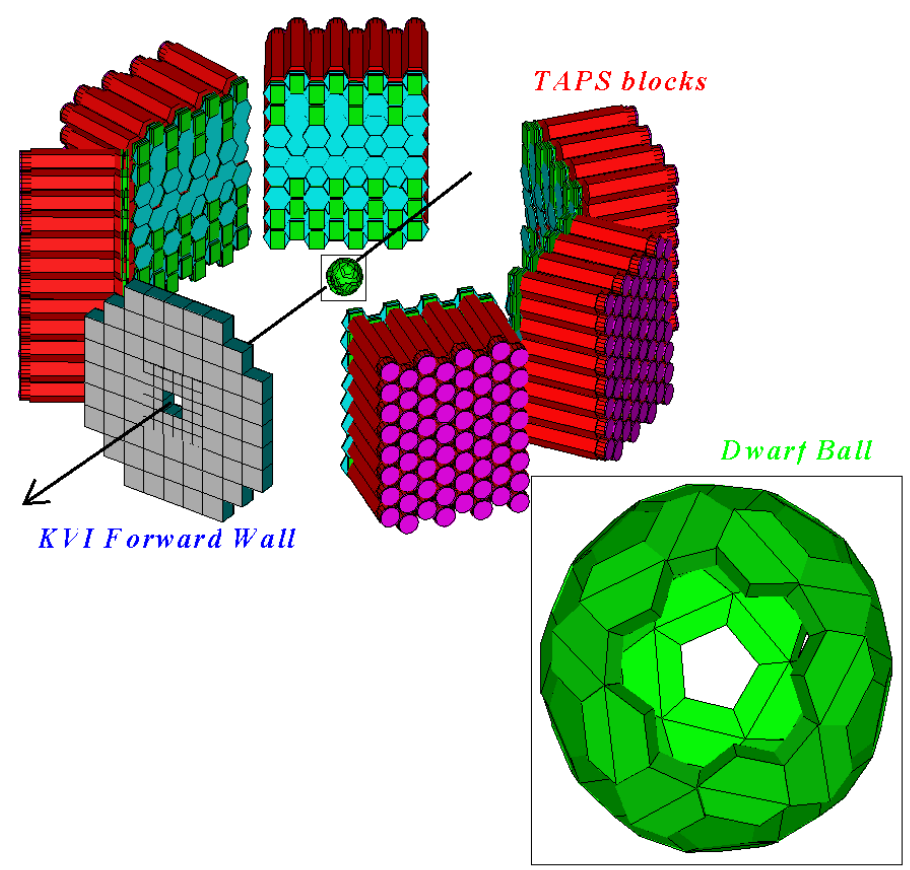

Fig. 1. GEANT layout of the detection system composed of TAPS electromagnetic calorimeter, and Dwarf-Ball and Forward Wall charged particle multidetectors.

The Washington University "Dwarf-Ball" (DB) [13] (composed of 64 BC400$\mathrm{CsI}(\mathrm{Tl})$ phoswich telescopes in the angular range $32^{\circ}<\theta<168^{\circ}$ covering around $76 \%$ of $4 \pi$ ) and the KVI's "Forward Wall" (FW) hodoscope [14 (92 NE102A-NE115 $\Delta \mathrm{E}$-E phoswich detectors in the forward angular range $2.5^{\circ}<\theta<25^{\circ}$ with a geometrical acceptance of $4 \%$ of $4 \pi$ ) allowed us to identify isotopically the different LCP produced $\left(p, d, t,{ }^{3} \mathrm{He}\right.$ and $\left.\alpha\right)$ and the charge of the IMFs up to that of the projectile by means of pulse-shape analysis techniques. 
The first reaction, ${ }^{36} \mathrm{Ar}+{ }^{197} \mathrm{Au}$, was thoroughly studied with the whole experimental setup (TAPS, DB and FW, see fig. 11) at low beam intensities; whereas the rest of the reactions were studied with a reduced setup (TAPS and FW) during a second set of high counting-rate runs.

\section{Experimental results}

Using the setup shown in fig. 1, more than $1.1 \cdot 10^{6}$ hard-photons for the four mentioned systems were collected out of $180 \cdot 10^{6}$ recorded events taking into account the different defined triggers. When dealing with nucleus-nucleus reactions, a first analysis of the experimental results usually considers inclusive (i.e. $A+B \rightarrow \gamma+X$, regardless of the particular final exit-channel) observables such as the shape of the photon spectra and the angular distributions (sections 3.1 and 3.2). Results from exclusive reactions, i.e. from selected $\gamma$-particles coincidence exit-channels, will be presented in section 3.3 .

\subsection{Hard-photon energy spectra}

In fig. 2 2 we show the photon energy spectra in the $N N$ CM frame for the heaviest $\left({ }^{197} \mathrm{Au}\right)$ and lightest $\left({ }^{12} \mathrm{C}\right)$ targets after correcting for the TAPS response function and after subtraction of the cosmic and $2-\gamma \pi^{0}$-decay backgrounds. A steeper slope in the region $E_{\gamma}=30-60 \mathrm{MeV}$ with respect to the flatter exponential falloff describing the high-energy part of the spectra above $E_{\gamma}=60 \mathrm{MeV}$ is clearly observed in the heaviest ${ }^{36} \mathrm{Ar}+{ }^{197} \mathrm{Au}$ system (as well as in the ${ }^{107} \mathrm{Ag}$ and ${ }^{58} \mathrm{Ni}$ targets, not shown here) but it is not present in the lightest ${ }^{36} \mathrm{Ar}+{ }^{12} \mathrm{C}$ reaction. In the three heavier systems, this excess of hard-photons clearly hinders the fit with a single exponential of the spectra above $30 \mathrm{MeV}$ as it has been commonly done in hard-photon studies [2]. To properly describe the measured spectra for the heavier systems we have, therefore, applied a sum of two exponential distributions corresponding, as proposed by [4, to a "direct" (coming from first-chance $p n \gamma$ collisions) and a "thermal" (secondary $p n \gamma$ ) hard-photon component respectively, with their corresponding weights:

$$
\frac{d \sigma}{d E_{\gamma}}=K_{d} e^{-E_{\gamma} / E_{0}^{d}}+K_{t} e^{-E_{\gamma} / E_{0}^{t}}
$$

The slopes of the direct component $\left(E_{0}^{d} \approx 20 \mathrm{MeV}\right)$ are between two to three times larger than the thermal ones $\left(E_{0}^{t} \approx 6-9 \mathrm{MeV}\right)$ and the contribution of thermal hard-photons represents a $15 \%-20 \%$ of the total hard-photon yield for the three heavier targets (Table I). In the small ${ }^{36} \mathrm{Ar}+{ }^{12} \mathrm{C}$ projectile-target combination, the resulting zone of participant nuclear matter does not have the necessary volume to achieve sufficient stopping and consequent thermalization and, hence, for that system pure direct bremsstrahlung clearly dominates the whole photon emission above $E_{\gamma}=30 \mathrm{MeV}$.

The slope parameters of the direct component follow the known linear dependence with the projectile energy per nucleon in the laboratory frame as expected 
Thermal hard-photons probing multifragmentation...
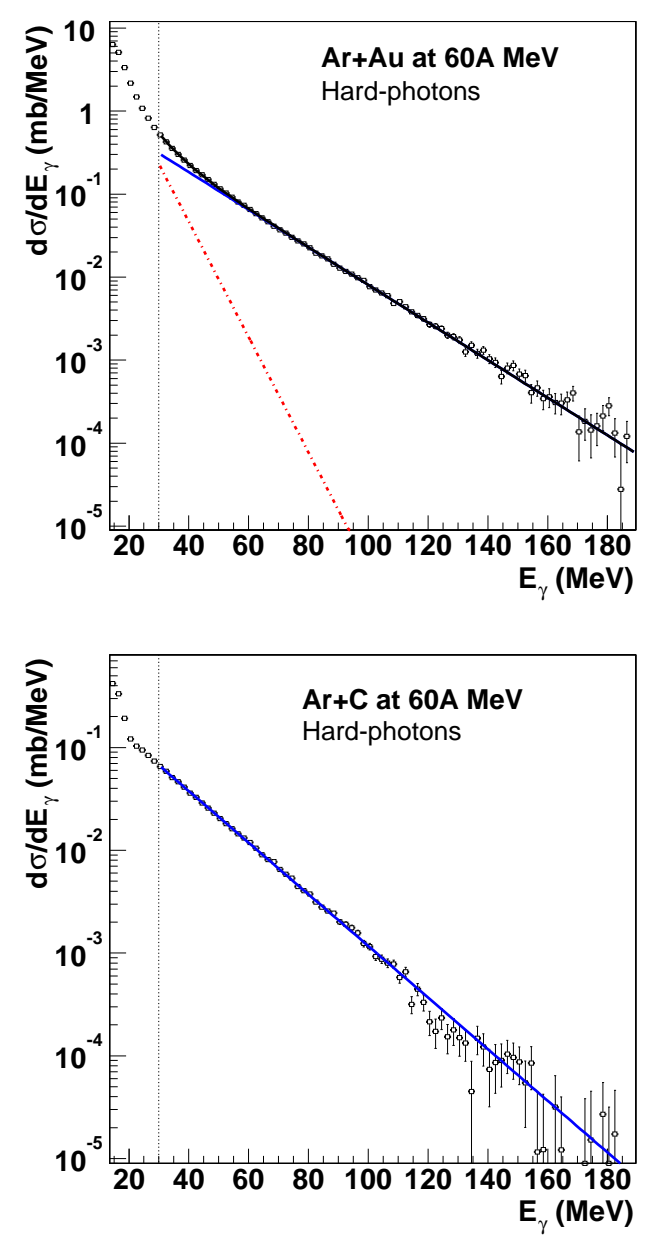

Fig. 2. Experimental hard-photon $\left(E_{\gamma}>30 \mathrm{MeV}\right)$ spectra measured in the $N N$ center-ofmass frame for the heaviest $\left({ }^{36} \mathrm{Ar}+{ }^{197} \mathrm{Au}\right.$, left side) and the lightest $\left({ }^{36} \mathrm{Ar}+{ }^{12} \mathrm{C}\right.$, right side) systems and fitted, according to equation (1), to the sum of two exponential distributions: a direct (solid line) and a thermal one (dashed line).

for pre-equilibrium emission in first-chance $N N$ collisions [2]. The thermal hardphoton slopes, at variance, scale with the available energy in the nucleus-nucleus center-of-mass (fig. 3), suggesting that they are emitted at a later stage of the reaction when the incident kinetic energy has been dissipated into internal degrees of freedom in the $A A$ system. 
Table 1. Ratios of thermal to total intensities, as well as direct and thermal slopes for the hard photons measured in the ${ }^{36} \mathrm{Ar}+{ }^{197} \mathrm{Au},{ }^{107} \mathrm{Ag},{ }^{58} \mathrm{Ni},{ }^{12} \mathrm{C}$ reactions at $60 \mathrm{~A} \mathrm{MeV}$. The available energies in the lab and in the AA CM frames for each reaction are also reported.

\begin{tabular}{cccccc}
\hline \hline System & $I_{t} / I_{\text {tot }}$ & $E_{0}^{d}(\mathrm{MeV})$ & $E_{0}^{t}(\mathrm{MeV})$ & $E_{C c}^{\text {lab }}(A \mathrm{MeV})$ & $E_{C c}^{A A}(A \mathrm{MeV})$ \\
\hline${ }^{36} \mathrm{Ar}+{ }^{197} \mathrm{Au}$ & $0.19 \pm 0.01$ & $20.2 \pm 1.2$ & $6.2 \pm 0.5$ & 55.5 & 7.3 \\
${ }^{36} \mathrm{Ar}+{ }^{107} \mathrm{Ag}$ & $0.15 \pm 0.01$ & $20.1 \pm 1.3$ & $6.1 \pm 0.6$ & 57.0 & 10.7 \\
${ }^{36} \mathrm{Ar}+{ }^{58} \mathrm{Ni}$ & $0.20 \pm 0.01$ & $20.9 \pm 1.3$ & $8.8 \pm 0.8$ & 58.1 & 13.7 \\
${ }^{36} \mathrm{Ar}+{ }^{12} \mathrm{C}$ & $0.00 \pm 0.05$ & $18.1 \pm 1.1$ & $0.0 \pm 0.5$ & 59.5 & 11.1 \\
\hline \hline
\end{tabular}

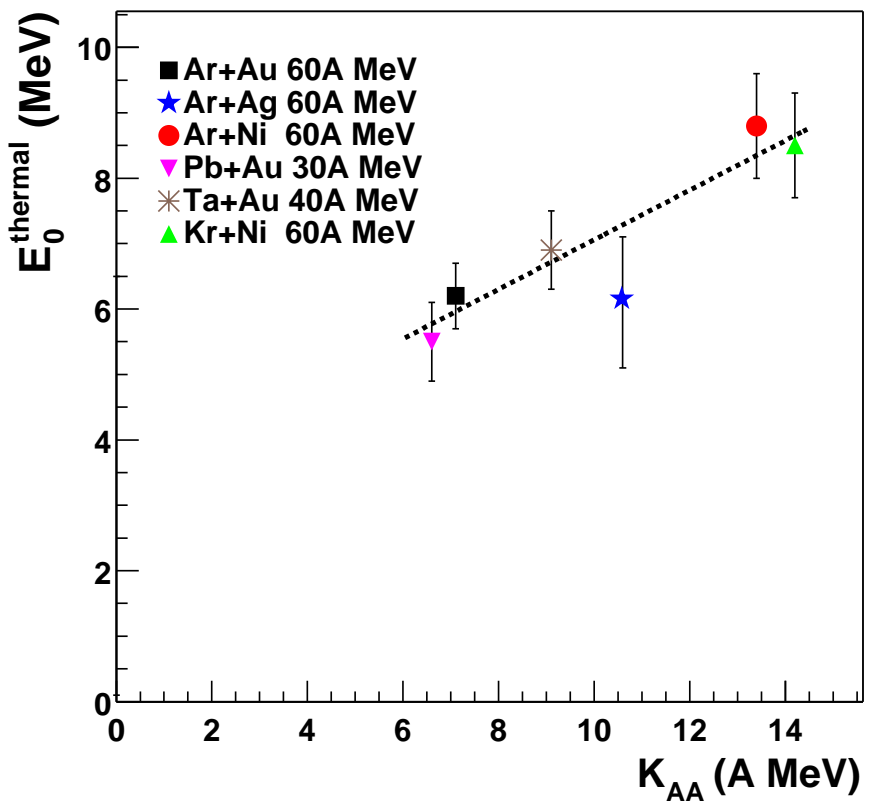

Fig. 3. Thermal hard-photon slope parameters, $E_{0}^{t}$, as a function of the (Coulombcorrected) nucleus-nucleus center-of-mass energy, $K_{A A}$, for the six systems studied in the TAPS campaign at KVI in 1997 and in the 1992 TAPS campaign at GANIL [6]. The dashed line is a linear fit to the data.

\subsection{Hard-photon angular distributions}

The interpretation of the second component of the hard-photon spectrum as being emitted during later stages of the reaction is confirmed by the study of the Doppler-shifted laboratory angular distributions. The angular distribution of bremsstrahlung photons emitted in heavy-ion reactions can be parametrized, in the 
frame of the emitting source, by an isotropic and an anisotropic dipolar term [2]. The measured angular distributions in the laboratory system contain an additional Lorentz-boost term, $Z=\gamma_{S}\left(1-\beta_{S} \cos \theta_{l a b}\right)$, depending on the velocity $\beta_{S}$ of the moving photon source:

$$
\left(\frac{d \sigma}{d \Omega}\right)_{l a b}=\frac{K}{Z\left(\beta_{S}\right)^{2}}\left[1-\alpha+\alpha \frac{\sin ^{2} \theta_{\gamma}^{l a b}}{Z\left(\beta_{S}\right)^{2}}\right] E_{0}^{d} e^{-30[\mathrm{MeV}] \cdot Z\left(\beta_{S}\right) / E_{0}^{d}}
$$

Above $\mathrm{E}_{\gamma}=60 \mathrm{MeV}$, the photon spectra are dominated by "pure" direct hardphotons for all four systems and their angular distributions are very well described with equation (2) assuming an emission from a source moving with the nucleonnucleon center-of-mass velocity $\left(\beta_{S} \approx \beta_{N N}=0.17\right)$. However, the average source velocities obtained from the laboratory angular distributions of the hard-photons with energies between $30 \mathrm{MeV}$ and $45 \mathrm{MeV}$ emitted in the three heavier reactions, are a factor $10 \%$ to $25 \%$ lower $\left(\beta_{S} \approx 0.14\right)$. The presence in this photon energy range of an important second bremsstrahlung component, issuing from later nucleon-nucleon collisions taking place within a slowly recoiling residue, induces this lowering of the average velocity of the hard-photon moving source. As a matter of fact, the hard-photon angular distributions above $30 \mathrm{MeV}$ (fig. (1) can be well reproduced with the distribution expected for the emission from a first-chance source with slope parameter $E_{0}^{d}$ moving with $\beta_{S}^{d} \approx \beta_{N N}$ plus a second-chance isotropic source with slope parameter $E_{0}^{t}$ and $\beta_{S}^{t} \approx \beta_{A A}$ (the ratios of thermal to direct intensities being fixed by those obtained from the energy spectra). Such an expression reads:

$$
\left(\frac{d \sigma}{d \Omega}\right)_{l a b}=\frac{K}{Z^{2}}\left[1-\alpha+\alpha \frac{\sin ^{2} \theta_{\gamma}^{l a b}}{Z^{2}}\right] E_{0}^{d} e^{-30[\mathrm{MeV}] \cdot Z / E_{0}^{d}}+\frac{K^{\prime}}{Z^{\prime 2}} E_{0}^{t} e^{-30[\mathrm{MeV}] \cdot Z^{\prime} / E_{0}^{t}}
$$

where $E_{0}^{d, t}$ are the slope parameters of the direct and thermal components in the source frame respectively, and $Z=1 / \sqrt{1-\left(\beta_{S}^{d}\right)^{2}} \cdot\left(1-\beta_{S}^{d} \cos \theta_{\gamma}^{l a b}\right)$ and $Z^{\prime}=1 / \sqrt{1-\left(\beta_{S}^{t}\right)^{2}} \cdot\left(1-\beta_{S}^{t} \cos \theta_{\gamma}^{\text {lab }}\right)$ are the Lorentz-boost factors corresponding to the direct and thermal source moving frames, $K, K^{\prime}$ are the normalization factors associated to the direct and thermal intensities $I_{d}$ and $I_{t}$ respectively, and $\alpha$ is the weight of the dipole component (only for the direct component). The values of the two sources velocities $\beta_{S}^{d}$ and $\beta_{S}^{t}$ as well as the intensity of the thermal component obtained with such a fit of the laboratory angular distributions are summarized in table 2 .

This result is consistent with direct hard-photons being emitted from the nucleonnucleon center-of-mass, and thermal hard-photons being emitted isotropically from a source moving with the nucleus-nucleus center-of-mass velocity. 


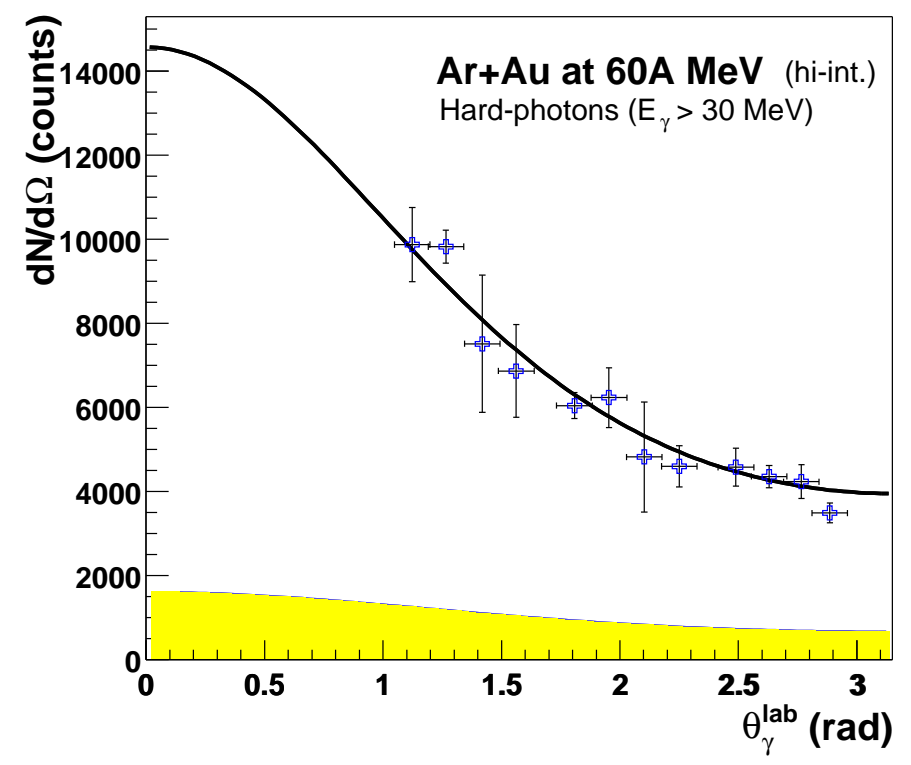

Fig. 4. Experimental angular distribution in the lab for hard-photons $\left(E_{\gamma}>30 \mathrm{MeV}\right)$ measured in the system ${ }^{36} \mathrm{Ar}+{ }^{197} \mathrm{Au}$ during the high counting-rate runs and fitted according to equation (3). The hatched region indicates the estimated contribution of thermal hardphotons, emitted isotropically from a source moving with $\beta_{A A}$.

Table 2. Direct and thermal hard-photon $\left(E_{\gamma}>30 \mathrm{MeV}\right)$ source velocities obtained from a fit of hard-photon laboratory angular distributions to equation (3).

\begin{tabular}{lccccc}
\hline \hline Reaction & $\beta_{S}^{d}$ & $\beta_{S}^{t}$ & $\alpha$ & $I_{t} / I_{\text {tot }}$ & $\chi^{2} / \nu$ \\
\hline${ }^{36} \mathrm{Ar}+{ }^{197} \mathrm{Au}$ & $0.17 \pm 0.02$ & $0.06 \pm 0.01$ & $0.0 \pm 0.0$ & $19 \% \pm 2 \%$ & 1.7 \\
${ }^{36} \mathrm{Ar}+{ }^{107} \mathrm{Ag}$ & $0.18 \pm 0.01$ & $0.09 \pm 0.01$ & $0.0 \pm 0.0$ & $19 \% \pm 2 \%$ & 0.3 \\
${ }^{36} \mathrm{Ar}+{ }^{58} \mathrm{Ni}$ & $0.18 \pm 0.03$ & $0.14 \pm 0.02$ & $0.0 \pm 0.0$ & $20 \% \pm 2 \%$ & 0.5 \\
${ }^{36} \mathrm{Ar}+{ }^{12} \mathrm{C}$ & $0.20 \pm 0.01$ & $0.00 \pm 0.05$ & $0.25 \pm 0.05$ & $0 \% \pm 2 \%$ & 1.1 \\
\hline \hline
\end{tabular}

\subsection{Exclusive results}

By selecting specific centrality channels by means of the measured chargedparticle multiplicity, we have been able to study as a function of impact-parameter the yield of different types of photons emitted in the ${ }^{36} \mathrm{Ar}+{ }^{197} \mathrm{Au}$ reaction:

1. (Mainly) Giant-Dipole-Resonance (GDR) photons, defined as photons with 
energies in the range $10 \mathrm{MeV}<\mathrm{E}_{\gamma}<22 \mathrm{MeV}$,

2. "Mixed" 1 ] thermal and direct hard-photons, in the range $30 \mathrm{MeV}<\mathrm{E}_{\gamma}<$ $45 \mathrm{MeV}$,

3. "Pure" direct hard-photons (i.e. photons with $\mathrm{E}_{\gamma}>60 \mathrm{MeV}$ ).

Thermal and direct bremsstrahlung photons are produced in very similar reaction channels that are clearly different to those that lead to GDR statistical photon emission as can be seen in the distribution of the photon multiplicities (gamma yield per nuclear reaction) versus the charged-particle multiplicity detected in the Dwarf-Ball (fig. 5). Thermal and direct hard-photon yields show a very similar dependence with impact-parameter, increasing a factor $\approx 10$ when going from peripheral $\left(M_{c p}=2\right)$ to semi-central $\left(M_{c p} \approx 10\right)$ reactions. They saturate in the region of semi-central and central $\left(M_{c p}=9-18\right)$ reactions, i.e. at the region of charged-particle multiplicities corresponding to impact parameters where the total overlapping of the incident ${ }^{36} \mathrm{Ar}$ nucleus inside the much larger ${ }^{197} \mathrm{Au}$ target nucleus takes place. In this region of impact-parameters, the mean number of $N N$ collisions saturates, i.e. $\left\langle N_{p n}\right\rangle_{b}=\left\langle N_{p n}\right\rangle_{\max }$, and hence so does the bremsstrahlung photon production. This confirms that $M_{\text {hard }-\gamma}$ is proportional to the number of participants and ultimately to the volume of the overlap region between projectile and target at a given impact-parameter.

The GDR photon yield, contrarily, clearly quenches for increasingly central reactions. Indeed, the yield of photons with $10 \mathrm{MeV}<\mathrm{E}_{\gamma}<22 \mathrm{MeV}$ is reduced roughly a factor 2 between $M_{c p}=7$ and $M_{c p} \approx 20$. This result confirms the significant quench of the GDR gamma yield observed experimentally for increasingly high excitation energies $\left(\epsilon^{\star}>3 \mathrm{~A} \mathrm{MeV}\right)$ in several systems [15, 16]. This quenching has been tentatively interpreted as a result of the loss of collectivity due to a change from ordered mean-field-driven motion to chaotic nucleonic motion. This interpretation seems to be nicely corroborated here.

\section{Summary}

Hard-photon production has been measured in coincidence with most of the reaction products in four different heavy-ion reactions $\left({ }^{36} \mathrm{Ar}+{ }^{197} \mathrm{Au},{ }^{107} \mathrm{Ag},{ }^{58} \mathrm{Ni},{ }^{12} \mathrm{C}\right.$ at $60 \mathrm{~A} \mathrm{MeV}$ ). The first results demonstrate the existence of a thermal bremsstrahlung component in the three heavier systems showing up in the region $\mathrm{E}_{\gamma}=30 \mathrm{MeV}-60$ $\mathrm{MeV}$ as a deviation from the flatter exponential fall-off describing the high-energy part of the spectra above $60 \mathrm{MeV}$. The thermal hard-photon yield accounts for about $20 \%$ of the total hard-photon yield for the heaviest targets ( $\mathrm{Au}, \mathrm{Ag}$ and $\mathrm{Ni}$ ) and is negligible for the ${ }^{36} \mathrm{Ar}+{ }^{12} \mathrm{C}$ system. The thermal slopes scale with the available center-of-mass energy in the nucleus-nucleus system as expected for a thermal

\footnotetext{
1) According to the ratio of intensities of the thermal component with respect to the total hard-photon yield above $30 \mathrm{MeV}$ in the ${ }^{36} \mathrm{Ar}+{ }^{197} \mathrm{Au}$ system (table $\mathrm{E}$ ), the relative proportion of thermal to direct bremsstrahlung photons in the range $30 \mathrm{MeV}<\mathrm{E}_{\gamma}<45 \mathrm{MeV}$ is 40:60 (thus the term "mixed" for this second selected type of photons).
} 


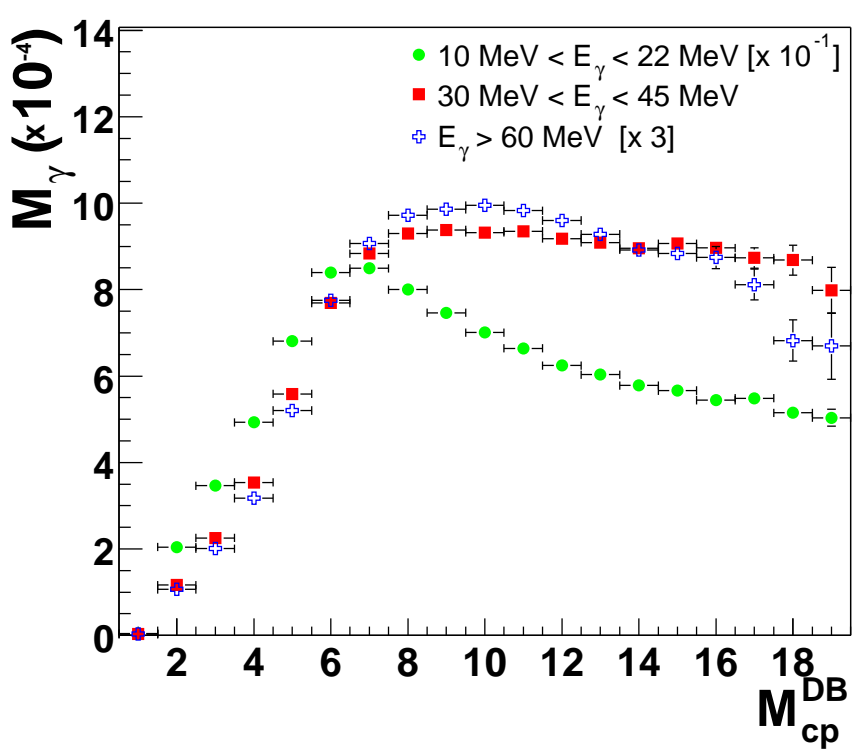

Fig. 5. Experimental photon yield per nuclear reaction, $M_{\gamma}$, as a function of the chargedparticle multiplicity, $M_{c p}^{D B}$, measured in the Dwarf-Ball for: (1) GDR photons, (2) thermal+direct hard-photons, and (3) "pure" direct hard-photons, emitted in ${ }^{36} \mathrm{Ar}+{ }^{197} \mathrm{Au}$ at $60 \mathrm{~A} \mathrm{MeV}$. (The photon multiplicity distributions in the region of the GDR-photons and of the direct hard-photons have been scaled to that of the thermal+direct hard-photons.)

process taking place after dissipation of the available energy into internal degrees of freedom over the whole system, whereas direct slopes scale with the available energy in first-chance $N N$ collisions. Source velocity analysis is consistent with a later isotropic emission process for the thermal component. The dependence of the thermal and direct hard-photon yields on impact parameter is very similar, confirming a common underlying bremsstrahlung mechanism for their production, significantly different than that of photons coming from the statistical decay of collective GDR excitations. The existence of such thermal bremsstrahlung emission is an unambiguous indication of the formation of a thermalized nuclear source during the reaction. The thermal slopes, which are correlated with the temperature of the excited nuclei [6, 17], become a good candidate variable for the determination of the temperature of the excited nuclear residues remaining after the first pre-equilibrium phase of the reaction, providing a unique information of the thermodynamical state of multifragmenting nuclear systems [17].

\section{References}

[1] W. Cassing, V. Metag, U. Mosel and K. Niita, Phys. Rep. 188 (1990) 363. 
Thermal hard-photons probing multifragmentation...

[2] H. Nifenecker and J. Pinston, Ann. Rev. Nucl. Part. Sci. 40 (1990) 113.

[3] R. Novotny, IEEE Trans. Nucl. Sci. NS-38 (1991) 379.

[4] G. Martínez et al., Phys. Lett. B349 (1995) 23.

[5] F.M. Marqués et al., Phys. Lett. B349 (1995) 30.

[6] Y. Schutz et al., Nucl. Phys. A622 (1997) 405.

[7] J.P. Bondorf et al., Phys. Rep. 257 (1995) 133.

[8] D.H. Gross, Phys. Rep. 279 (1997) 119.

[9] J. Aichelin, Phys. Rep. 202 (1991) 233.

[10] Hirschegg '99, "Multifragmentation", Proc. Int. Workshop XXVII on Gross Prop. of Nuclei and Nuc. Excitations, Hirschegg, Austria, Jan. 1999, ed. H. Feldmeier et al., GSI, Darmstadt, 1999.

[11] R. Ortega, these Proceedings.

[12] F.M. Marqués et al., Nucl. Inst. and Meth. A365 (1995) 392.

[13] D. Stracener et al., Nucl. Inst. and Meth. A294 (1990) 485.

[14] H. Leegte et al., Nucl. Inst. and Meth. A313 (1992) 26.

[15] J.J. Gaardhoje, Ann. Rev. Nucl. Part. Sci. 42 (1992) 483.

[16] T. Suomijärvi et al., RIKEN Symposium on Dynamics in Hot Nuclei, March 13-14, 1998, IPNO DRE 98-09.

[17] D.G. d'Enterria, PhD thesis, U.A. Barcelona and U. Caen, Mars 2000. 